\title{
Three-Dimensional Imaging of Corrosion Mechanisms in Polymer Electrolyte Fuel Cells by Scanning Transmission Electron Tomography
}

\author{
J.K. Hyun, ${ }^{*}$ Z.Y. Liu, ${ }^{* *}$ and D.A. Muller*** \\ * Department of Physics, Cornell University, Ithaca, NY 14853 \\ ** General Motors- Fuel Cell Research Laboratory, Honeoye Falls, New York, 14472 \\ *** Department of Applied and Engineering Physics, Cornell University, Ithaca, NY 14853
}

Polymer Electrolyte Membrane Fuel Cells (PEMFC) are promising candidates for electricity generation in automobiles. A typical PEMFC unit consists of a central component known as the membrane electrode assembly (MEA). Within the MEA, the cathode electrode is responsible for the reduction of proton with oxygen to produce water. Normally, high surface-area carbon such as Vulcan or Ketjen black is used to support the platinum $(\mathrm{Pt})$ catalysts for the cathode electrode. Performance degradation of the MEA can occur due to corrosion of the carbon supports during transient start/stop operations, or localized hydrogen-fuel starvation from flow maldistribution [1]. Visualization of the morphology and structure of corroded carbon/Pt systems to identify early failure sites is therefore a necessary first-step in understanding the mechanism and structural dynamics of carbon corrosion [2].

We examine the three-dimensional structure of corroded carbon/Pt systems using low-angle annular-dark-field (LAADF) STEM tomography. A $490 \mathrm{~mm}$ camera length for the LAADF detector was necessary to enhance the contrast between the weakly scattering carbon and $\mathrm{SiO}_{\mathrm{x}}$ background. Samples were prepared by scraping powder off from the central corroded region of an aged MEA (Gore 5700 MEA, W. L. Gore \& Associates, USA), dispersed in water by sonication, and loaded onto holey $\mathrm{SiO}_{\mathrm{x}}$ film $\mathrm{Cu}$ TEM grids. A tilt series of 71 images was acquired from -68 degrees to 72 degrees at $2^{\circ}$ increments in a $200 \mathrm{kV}$ FEI TF-20 TEM/STEM system and using a Fischione model 2020 advanced tomography holder. The magnification for each image was $320 \mathrm{kx}$ taken with 512 x 512 pixels, corresponding to $0.7 \mathrm{~nm}$ per pixel.

The carbon oxidation results in voids inside the carbon particles (Fig. 1a). This is in stark contrast to non-corroded carbon supports (Fig. 1b). The inside-out corrosion is likely because the outer-layer of the particle has a well-organized crystallite construction [3], and is structurally more resistant to oxidation. Oxidation channels linking outside to interior are also resolved in the 3D analysis (Fig. 2), reflecting the paths through which oxidation attacks have taken place.

The structural units for carbon black can be categorized as primary particle, aggregate and agglomerate [4]. Primary particles refer to single carbon particles, and aggregates describe primary particles fused together through covalent bonds. Slices through the reconstructed tomogram (Fig. 3) show that the widths of the carbon wall stay independent of size of the carbon particle. In addition, the interconnectivity of voids amongst the carbon particles suggests that carbon aggregates rather than individual particles corrode as a unit. These conclusions are important for designing more accurate microstructual models used in corrosion mechanism studies. 
References

[1] R. Macharia et al., J. Electrochem. Soc. 152 (2005) A970

[2] Z.Y. Liu et al., J. Electrochem. Soc. 155 (2008) B979

[3] F.A. Heckman and D.F. Harling, Rubber Chem. Technol. 39 (1996) 1

[4] W.M. Hess and C.R. Herd, Carbon Black, $2^{\text {nd }}$ ed. (London, 1993) 106

[5] This project has been supported by NSF EEC-0117770, NYSTAR C020071, and the Semiconductor Research Corporation and facilities MRSEC DMR-0520404 funding
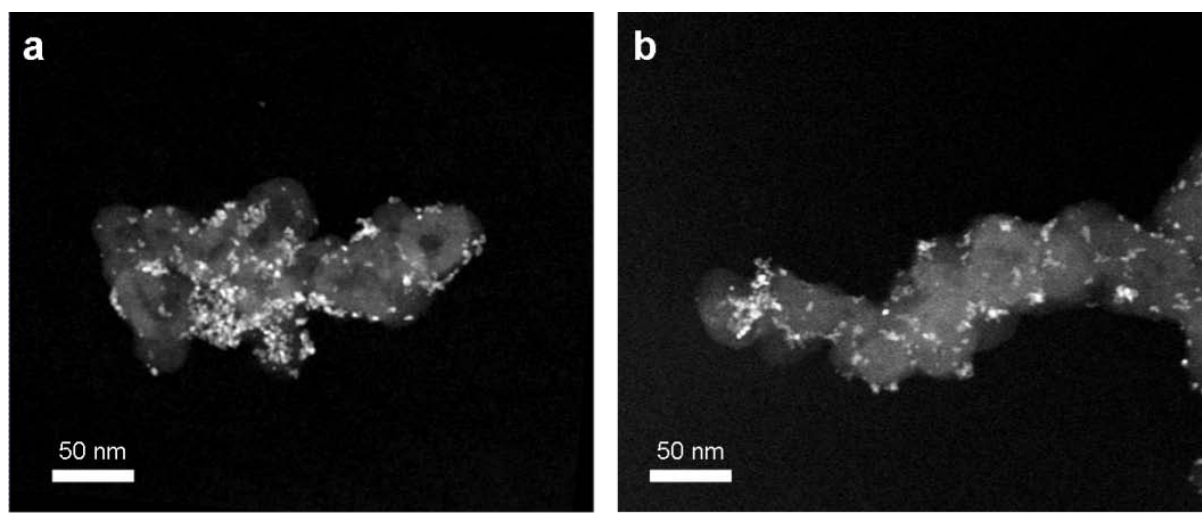

FIG. 1. LAADF-STEM images of (a) Pt/corroded carbon support and (b) Pt/non-corroded carbon support.

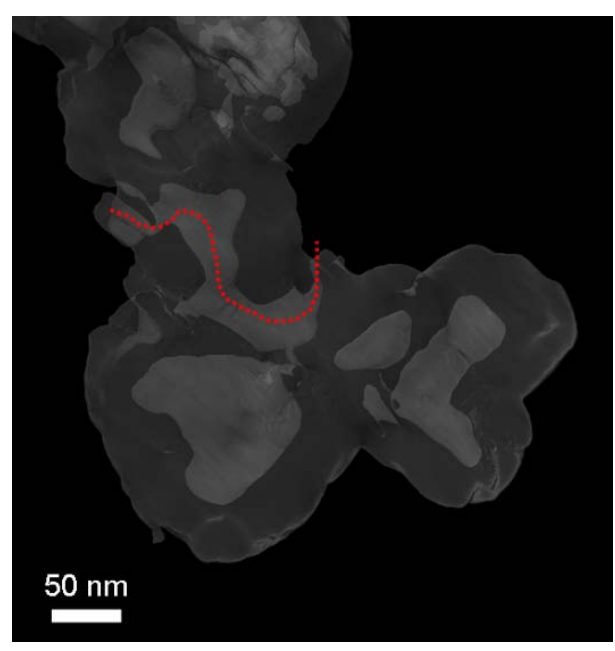

FIG. 2. Reconstructed corroded carbon support without the $\mathrm{Pt}$ catalysts for clearer visualization of the void interconnectivities. The dotted red curve illustrates an oxidation channel through the structure.
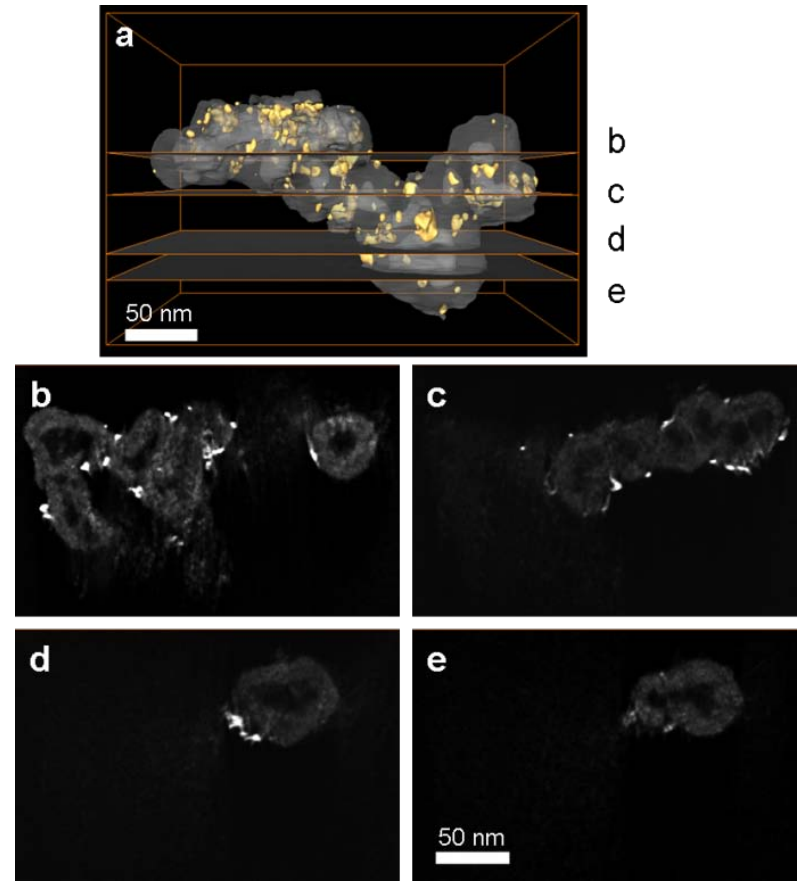

FIG. 3. (a) The Pt/corroded carbon tomogram. (b-e) Surface slices cut orthogonally through the tomogram. Intensities in the slices are scaled linearly. 